\title{
Determination of the Effect of Chalcogen Replacement on the Interaction Site and Transition State of the Substituted Analogues of Formaldehyde with Aldehyde Oxidase: A Density Functional Theory Approach
}

\section{Tadege Belay}

South Gonder (Debre Tabor University), Debre Tabor, Ethiopia; e-mail: tadege21@ gmail.com

\begin{abstract}
Aldehyde oxidase (AO) enzyme is known to oxidize aldehydes. One of the aldehydes, formaldehyde, is known to inhibit xanthine oxidase as it turns over. However, there is no reported data whether it behaves the same when it reacts with aldehyde oxidase. Similarly, the effect of chalcogen replacement on nucleophilic reaction and charge density distribution on the substituted analogs of formaldehyde and their behavior during catalysis has never been studied. Therefore, the research is intended to probe the most tractable substrate that interacts to the reductive half-reaction active site of AO. Therefore, a density functional theory of the B3LYP correlation functional formalism (DFT-B3LYP) methods was used to generate several parameters from the electronic structure calculations. Accordingly, the higher percentage (\%) contribution to HOMO and energy barrier $(\mathrm{kcal} / \mathrm{mol})(0.099,-7.185040 \mathrm{E}+04)$ makes formaldehyde as the favored substrate for aldehyde oxidase, compared to thioformaldehyde $(-0.245,-2.745113 \mathrm{E}+05)$ and selenoformaldehyde $(-0.175,-1.529992 \mathrm{E}+06)$, respectively. In addition, the transition state structures for the active site bound to formaldehyde (ACT-FA), thioformaldehyde (ACT-THIO FA), and selenoformaldehyde (ACT-SELENO FA), respectively, were confirmed by one imaginary negative frequency (S-1) $(-328.44$, -430.266 , and -624.854).
\end{abstract}

Received: March 25, 2019; Accepted: April 27, 2019

Keywords and phrases: aldehyde oxidase, formaldehyde, thioformaldehyde, selenoformaldehyde, chalcogen, oxidative hydroxylation, DFT-B3LYP.

Copyright (C) 2019 Tadege Belay. This is an open access article distributed under the Creative Commons Attribution License, which permits unrestricted use, distribution, and reproduction in any medium, provided the original work is properly cited. 


\section{Introduction}

Aldehyde oxidase (AO) is one of the xanthine oxidase family enzymes that catalyze the oxidative hydroxylation reactions [1]. AO is also widely distributed throughout the animal kingdom, such as rat, bovine, rabbit, baboon, guinea pig and monkey [2]. AO is found in the liver, kidney, lung, spleen, stomach, muscle and heart [2]. AO is predominant in the liver whereas $\mathrm{XO}$ is present mainly in the lactating mammary gland and primarily in cow's milk $[2,3,4]$.

The mechanism of action of aldehyde oxidase behaves similarly to xanthine oxidase due to their similarity in structure and molecular properties [4]. However, both enzymes show a different substrate and inhibitor specificities $[4,5]$. They catalyze the oxidation of a wide range of $\mathrm{N}$-heterocycles and aldehydes to the corresponding carboxylic acids [4]. Although the hydroxylation of aldehyde and purine substrates takes place at the molybdenum site, in both $\mathrm{AO}$ and $\mathrm{XO}$, the former is known to turn over aldehydes more efficiently. On the other hand, purines such as hypoxanthine and xanthine are good substrates for $\mathrm{XO}$, but poor substrates for AO [5]. Although all aldehydes are bona fide substrates of aldehyde oxidase, some aldehydes are known to be substrates and some inhibitors of XO.

As mentioned above, aldehyde oxidase is known to catalyze the hydroxylation of a wide range of substrates, including aliphatic and aromatic aldehydes, in addition to charged and uncharged heterocyclic compounds [4]. However, this review is limited to aldehydes, mainly aliphatic aldehydes.

Formaldehyde is one of the simplest aldehydes which undergoes nucleophilic addition reactions and is the bona fide substrate for aldehyde oxidase. Several theoretical studies employing the density functional theory are expected to be carried out for the catalytic oxidation of formaldehyde with molybdenum cofactor (Moco) [6]. However, the effects of chalcogen replacement on the interaction site and transition state for the catalytic oxidation of formaldehyde are not studied. One of the aims of the study is to illustrate the effects of chalcogen replacement on the interaction site and transition state of the substituted analogs of formaldehyde.

The chalcogen elements, such as oxygen, sulfur, and selenium-containing aldehydes are involved in a wide range of essential biological functions. All three elements are constituents of functional groups in biomolecules that participate in redox reactions [9]. 
Among these, formaldehyde is widely used and extensively studied because of its importance in various metabolic processes. However, the behavior of the interaction site during chalcogen replacement and their effect on the transition state structure of the substituted analogs of the substrate (FA) were not studied.

Understanding the nature of reacting species (active sites and substrates) (Figure 2.1) and their electrophilic and nucleophilic properties is the preliminary circumstances for the reaction to take place.

In order to achieve the desired objectives, computation approach is designed. Thus, the computation approach helps to evaluate the electronic structure and wave function descriptions of the geometries obtained in the interaction site. All computation calculations, for the geometries shown in Figure 2.1, are carried out by applying density functional theory (DFT) of the B3LYP (Becke3-Lee-Yang-Parr) correlation functional formalism (DFT-B3LYP) methods [6].

Upon completion of this work, key questions regarding the effect of chalcogen replacement on the interaction site and transition state, and the events taking place at the transition state are expected to be answered. The wave function and bonding descriptions provide insight into how electrons are transferred between the active site and substrate before or after the formation of $\mathrm{S}_{\mathrm{Mo}}-\mathrm{H}_{\mathrm{RH}}$ bond. The electronic energies are also given insight into the performance of the reactions. The research would provide a mechanistic insight towards the synthesis of new compounds, catalysts, and drugs. Generally, the theoretical work is expected to relate electronic structure contributions to enzymatic catalysis and provide a deeper mechanistic insight into the enzyme-catalyzed reactions. The ultimate goal of this work is to recognize the effect of chalcogen replacement on the interaction site and transition state to probe a plausible reaction mechanism.

\section{Materials and Methods}

\subsection{Materials}

The software packages that were used for the completion of the study were GaussView 3.0 (Gaussian, Inc., Pittsburgh PA, USA), Gaussian 03W version 6.0 (Gaussian, Inc., Wallingford, CT, U.S.A), AOMix 2011/2012 version 6.6 (Centre for Catalysis Research and Innovation, the University of Ottawa), Chem Draw Ultra, version 8.0 (Cambridge soft corp., Cambridge, MA, USA), Dell computer Optiplex 780 
model, 2011 (Dell, Inc., Wilhile Sdhbhd., Penang, Malaysia), Microsoft Excel 2003/2007 and word 2007 (Microsoft, Inc., Redmond, Washington, U.S.A). The Chem Draw Ultra, version 8.0 was used to draw/sketch the structures. The structures (geometries) were constructed and input geometries for geometry optimization were prepared using GaussView 3.0 software package. In addition, the GaussView software was also used to compute the bond distances from the output geometries of the optimized structures and visualize pictorial view of frontier orbitals generated from checkpoint files. All computation calculations were performed using Gaussian 03W (version 6.0) program software package. The molecular orbital analyses for the constituent chemical fragments were performed using AOMix 2011/2012 version 6.6 software package. These analyses were performed from the output files obtained from the single point energy calculations. Finally, Microsoft Excel, PowerPoint and Word were used for data handling, and research writes up. All calculations and write-up were carried out on Dell Computer.

\subsection{Methods}

\subsubsection{General computation methods}

Electronic structure calculations were performed to generate Mulliken atomic charges, total electronic energies, wave function description, and bonding descriptions. All computation calculations were carried out by applying the density functional theory (DFT) method of the B3LYP (Becke3-Lee-Yang-Parr) correlation functional formalism (DFT-B3LYP) [6] using the Gaussian 03W (version 6.0) program software packages [7]. The 6-31G (d', p') basis set with a polarization function was applied for non-metal atoms (C, H, O, and S). Similarly, the LANL2DZ (Los Alamos National Laboratory 2 Double Zeta) basis set and LANL2 effective core potentials were applied for Mo atoms [6, 8]. The DFT method employing the B3LYP level of theory was applied on the truncated analog of the reductive half-reaction active site of aldehyde oxidase bound to formaldehyde shown in Figure 2.1. In all types of linear transit scan, geometry optimization, frequency, single point energy, and transition state search calculations, the "\# b3lyp gen pseudo=read \#p gfinput pop=full gfprint" general keywords and job type were used. In all calculations, the general job type and key words were used by applying other key words relevant to the respective calculations. 


\subsubsection{The effect of chalcogen replacement on the initial and catalysis stages}

\subsubsection{The effect of chalcogen replacement on the interaction site}

The geometries of the active site (left panel), formaldehyde (FA) (middle panel), and active site bound to FA (right panel) were prepared as input files using GaussView 03 software packages, by adjusting all bonding parameters to the crystal structure [10], such as bond distance, bond angle, and dihedral angle. The substrates, formaldehyde, thioformaldehyde, and selenoformaldehyde, were optimized in order to identify the effect of chalcogen replacement on the electron deficient carbon. The substrates bound to the reductive half-reaction active site (right panel) were also optimized. The free substrates and substrate bound to the reductive reaction active site, shown in Figure 2.1, were optimized by applying "opt iop $(6 / 7=3)$ " keyword to the general job type and keywords mentioned in the preceding section. The magnitude of the Mulliken atomic charges on the carbon atom and the bond distances were assembled from the optimized geometries. A single point energy calculation was performed for free substrates and substrate-bound intermediates by applying "iop $(3 / 33=1)$ " keyword to the general job type described in the preceding section. The percentage contribution of the carbon atoms and the bond orders were also assembled in order to analyze the behavior of the interaction site.

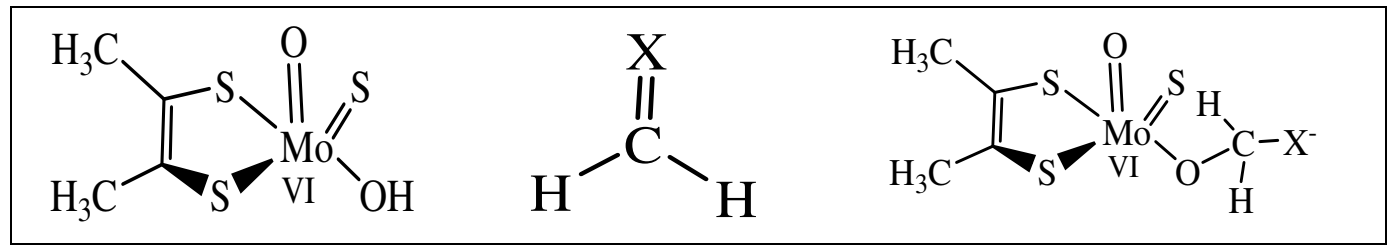

Figure 2.1. The geometries shown in the left, middle and right panels, respectively, represents the truncated form of the reductive half-reaction active site structure derived from crystal stricture of Desulfovibro gigas Aldehyde Oxidase (AO), the free substrates [formaldehyde $(\mathrm{X}=\mathrm{O})$, thioformaldehyde $(\mathrm{X}=\mathrm{S})$, and selenoformaldehyde $(\mathrm{X}=\mathrm{Se})$ ], and the active site bound to free substrates.

\subsubsection{The effect of chalcogen replacement on the transition state structures}

The transition state structure was located using a direct frequency calculation. The substrate-bound active site was constructed by placing the substrate " $\mathrm{H}$ or $\mathrm{H}_{\mathrm{RH}}$ " in between the active site sulfido terminal ("S or $\mathrm{S}_{\mathrm{Mo}}$ ") and the interaction site of the substrate or substrate carbon (" $\mathrm{C}_{\mathrm{RH}}$ "), as shown in Figure 2.2. In order to locate the 
transition state structure, direct frequency calculations were performed on formaldehyde (FA), thioformaldehyde (THIO FA), and selenoformaldehyde (SELENO FA) bound to the reductive half-reaction active site of AO.

In the direct frequency calculation, the frequency of a given structure was calculated without applying any constraint. The structures were calculated by applying "opt freq" keywords to the general keywords and job type mentioned in sub-section 2.2.1. In addition to the transition state structure (TS), the two structures (pre- and post-transition state structures) were optimized.

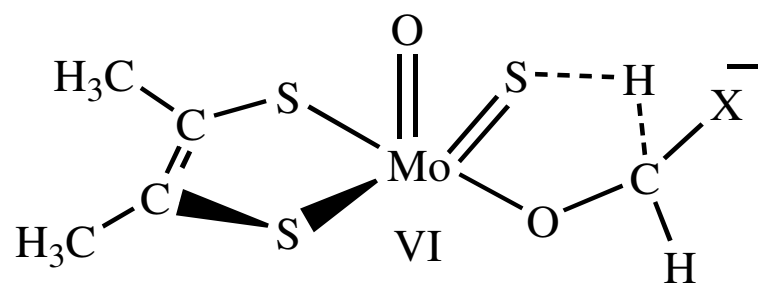

Figure 2.2. The general model structure used for the transition state structure search. The tetrahedral transition state structures were modeled by placing the substrate bound hydrogen $\left(\mathrm{H}_{\mathrm{RH}}\right)$ between the sulfido $\left(\mathrm{S}_{\mathrm{Mo}}\right)$ terminal and the substrate carbon $(\mathrm{CRH})$ center. In this structure, $\mathrm{X}$ was respectively $(\mathrm{O}, \mathrm{S}$, or $\mathrm{Se})$.

The pre-transition state structure $(\mathrm{CH})$ resembled substrate bound (structure near to substrate-bound) where the substrate $\left(\mathrm{H}_{\mathrm{RH}}\right)$ was bound to the interaction site of the substrate $\left(\mathrm{C}_{\mathrm{RH}}\right)$. The post-transition state structure $(\mathrm{SH})$ resembled product bound where the substrate $\left(\mathrm{H}_{\mathrm{RH}}\right)$ was bound to the sulfido terminal $\left(\mathrm{S}_{\mathrm{Mo}}\right)$ of the active site. The two structures were optimized by adding the constraint and applying "opt=moderedundant" keywords to the general job type. The input files for the three structures $(\mathrm{CH}$, TS, and $\mathrm{SH})$ of FA, THIO FA, and SELENO FA bound to the reductive half-reaction active site of AO, respectively, were prepared by fixing $\mathrm{S}_{\mathrm{Mo}}-\mathrm{H}_{\mathrm{RH}}$ bond distance at $2.26748 \AA$, $2.04097 \AA$, and $1.36097 \AA$. The charge and multiplicity assigned for the three structures were (-2) and (1), respectively.

All calculations were carried out at oxidation state (VI) for Mo $\left(\mathrm{Mo}^{\mathrm{VI}}\right.$ ) and constant charge and multiplicity, respectively, whole numbers (-2) and (1). The Mulliken atomic charges, the frequency value, electronic energy, bond order and the percentage 
contribution of the molybdenum metals to the highest occupied molecular orbital (HOMO) were generated from optimization and single point energy calculation, in order to analyze the effect of chalcogen replacement on the transition state structure.

\section{Results and Discussion}

\subsection{The effect of chalcogen replacement on the initial and catalysis stages}

As mentioned above, understanding the nature of reacting species (active sites and substrates) and their electrophilic and nucleophilic properties were the preliminary circumstances for the reaction to take place. As described earlier, formaldehyde was one of the simplest aldehydes which undergo nucleophilic addition reactions and was the bona fide substrate for aldehyde oxidase. In order to understand if the analogs of formaldehyde (chalcogen replaced) behave the same as formaldehyde and their effect on the interaction site and transition state, several parameters were used. These parameters were used to identify the relative susceptibility of the interaction sites to nucleophilic attack, upon chalcogen replacement.

\subsubsection{The effect of chalcogen replacement on the interaction site using total energy}

The energy values for free substrates (FA, THIO FA and SELENO FA) were found to $-7.185040 \mathrm{E}+04 \mathrm{Kcal} / \mathrm{mol},-2.745113 \mathrm{E}+05 \mathrm{Kcal} / \mathrm{mol}$, and -1.529992E+06 Kcal $/ \mathrm{mol}$, respectively. Therefore, FA was energetically unstable and labile to interact with the active site to form the Michaelis-Menten type complex. In addition, the energy values for FA and its chalcogen analog bound to the active site (AO) also followed the same trend and thus reflected the effect of stability of substrate-bound intermediates on the activation energy of the transition state. The higher the energy barrier of the transition state was due to the lesser reactivity of substrates and the more stable starting complex with stronger electrostatic interaction between substrates and the active site [6].

3.1.2. Understanding the effect of chalcogen replacement on the interaction site using Mulliken charges, percentage composition, and band gap energies

As shown in Figure 3.1, the Mulliken atomic charge on the carbon atom of FA was more electropositive (0.098894 a.u) than THIO FA (-0.245265 a.u) and SELENO FA $(-0.175047$ a.u). Unlike to this carbon atom, the remaining carbon atoms of chalcogen analogs were shown to bear partial negative charges. Hence, the electron deficient carbon atom of formaldehyde was less susceptible to nucleophilic attack to the 
catalytically liable equatorial oxygen of the active site than thioformaldehyde and selenoformaldehyde in order to form (Enzyme)-[Mo $\left.{ }^{\mathrm{VI}}-\mathrm{O}-\mathrm{C}_{\mathrm{CH}}\right]$ ) complex. However, THIO FA and SELENO FA were less liable to interact to the active site as a result of the electrostatic force of repulsion developed between them.

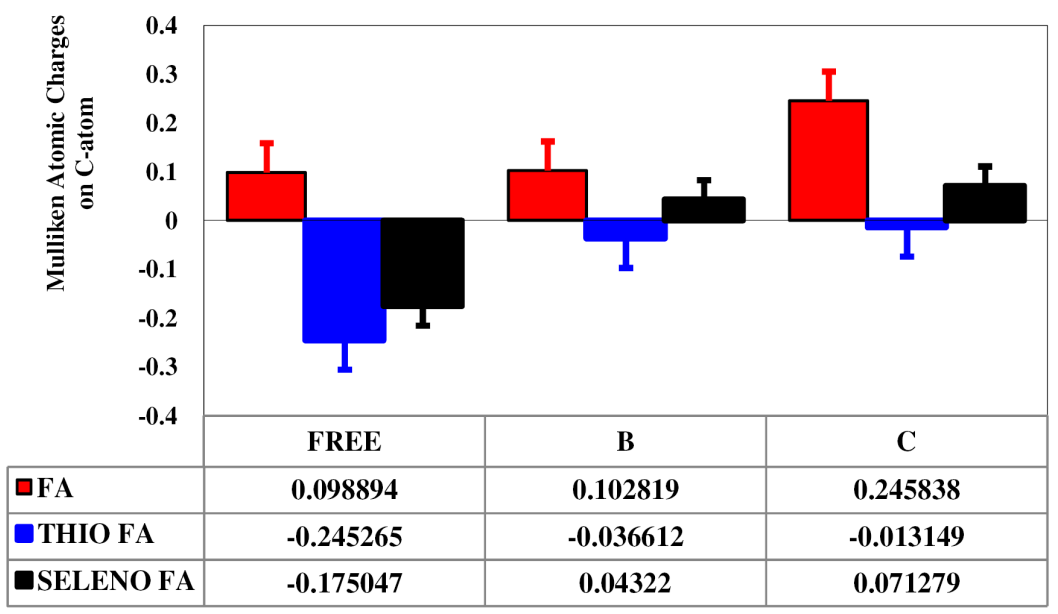

Figure 3.1. The Mulliken atomic charges on carbon atom obtained from geometry optimization of the free substrate structures [formaldehyde (FA), thioformaldehyde (THIO FA), and selenoformaldehyde (SELENO FA)], substrate bound to the truncated reductive half-reaction active site of AO (ACT-FA, ACT-THIO FA, and ACT-SELENO FA) structure (B), and substrate bound to the truncated reductive half-reaction active site of AO transition state (ACT-FA, ACT-THIO FA, and ACT-SELENO FA) structure (C). The 1st (red), 2nd (blue), and 3rd (black) bars (from left to right), respectively, indicated the Mulliken atomic charges on $\mathrm{C}$-atom for free substrates and substrate-bound active site.

Similarly, the percentage contribution of the carbon atoms to the highest occupied molecular orbitals (HOMOs) for C-atom of FA and its chalcogen analog were shown in Figure 3.2. The $\%$ contribution of the carbonyl carbon atom of the chalcogen family decreases down a group. The \% contribution to HOMO for C-atom of FA was 32\% greater than THIO FA and 39\% than SELENO FA. This indicated the most likely electron flows from the HOMO of FA to LUMO of the active site (Figure 2.1) than THIO FA and SELENO FA in order for the Michaelis-Menten type (Enzyme)-[Mo ${ }^{\mathrm{VI}}-\mathrm{O}-$ $\mathrm{C}_{\mathrm{CH}}$ ]) complex to be formed. On the contrary, the band gap energy (LUMO-HOMO gap) of SELENO FA was less than THIO FA and FA (Table 3.1). 


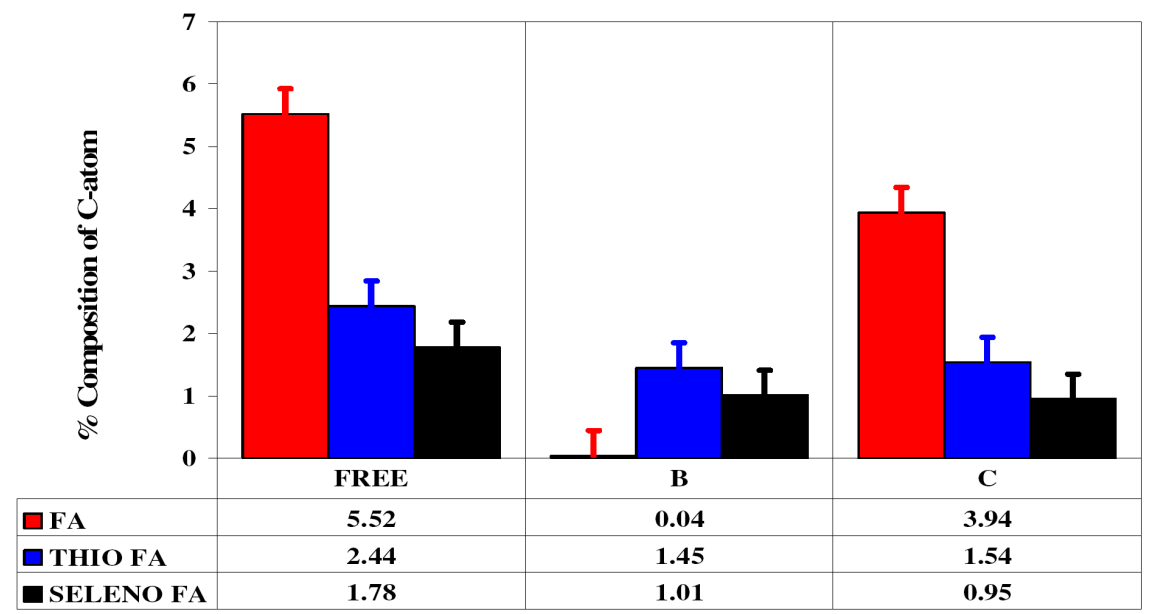

Figure 3.2. The $\%$ composition to the highest occupied molecular orbital (HOMOs) of carbon atom obtained from single point energy and AOMix calculations.

Table 3.1. The band gap energies (ev) of FA and its chalcogen analog obtained from AOMix calculation.

\begin{tabular}{lccc}
\hline \multirow{2}{*}{ Free Substrate } & \multicolumn{3}{c}{ Energy (ev) } \\
\cline { 2 - 4 } & HOMO & LUMO & Band gap \\
\hline \multirow{2}{*}{ FA } & -7.28 & -1.15 & 6.13 \\
THIO FA & -6.34 & -2.58 & 3.76 \\
SELENO FA & -5.98 & -2.72 & 3.26 \\
\hline
\end{tabular}

A molecule with a low energy gap could be a soft molecule due to its ability to be more polarizable and association with high chemical activity and low kinetic stability [11]. As the band gap energy $(\Delta \mathrm{E})$ decreased, the reactivity of the molecule was shown to increase leading to an increase in the percent ionization energy (\% IE) of the molecule. As a result, the lower energy gap of SELENO FA revealed that the energy required to remove an electron from the last occupied orbital was lower than FA and THIO FA. Therefore, the electrons could have been easily transferred from HOMO to LUMO orbital. This reflected the powerful reactivity nature of selenoformaldehyde compared to the remaining chalcogen analog. According to the principle of electrophilic /nucleophilic reaction and thermodynamics principles, formaldehyde was the tractable substrate to interact with the reductive half-reaction active site of AO. 
Moreover, the change in Mulliken atomic charge $(\Delta \mathrm{q})$ and $\%$ contribution of carbon for substrate bound (B) and transition state (C) structures were shown in Figures 3.1 and 3.2, respectively. The increase in Mulliken atomic charge on the carbonyl carbon atom (Figure 3.1), during the binding and catalysis stages of the reductive half-reactions, was due to the removal of bonding electrons (the breaking of $\mathrm{H}_{\mathrm{RH}}{ }^{-} \mathrm{C}_{\mathrm{RH}}$ and formation of $\mathrm{S}_{\mathrm{Mo}^{-}}$ $\mathrm{H}_{\mathrm{RH}}$ bonds at the transition state). The increase in \% contribution of C-atom (Figure 3.2) was due to the donation of electrons from the equatorial oxygen $\left(\mathrm{O}_{\mathrm{eq}}\right)$ to the substrate $\mathrm{C}$ atom $\left(\mathrm{C}_{\mathrm{RH}}\right)$. This was due to the interaction of HOMO of $\mathrm{O}_{\mathrm{eq}}$ and LUMO of $\mathrm{C}_{\mathrm{RH}}$ where the HOMOs acted as an electron pair donor and LUMOs as an electron pair acceptor. The energy of the highest occupied molecular orbital ( $\left.\mathrm{E}_{\mathrm{HOMO}}\right)$ measured the tendency towards the donation of an electron by a molecule [11]. The highest values of $\mathrm{E}_{\mathrm{HOMO}}$ were due to the tendency of the molecule to donate electrons to appropriate acceptor molecules with low energy and empty molecular orbital. The $(\Delta \mathrm{q})$ (Figure 3.1 ) and $\%$ contribution of C-atom (Figure 3.2) of structure (B) and (C) with respect to free (unbound) substrates decreases during the course of the reaction. Particularly, the \% contribution to HOMO for the carbonyl C-atom of formaldehyde was higher as compared to thioformaldehyde and selenoformaldehyde. Therefore, formaldehyde is believed to be liable to interact with the active site as a result of the better HOMO and LUMO interactions.

The $\% \mathrm{Mo}_{\mathrm{dxy}}$ to HOMO on the substrate bound intermediate (B) was lower than the transition state structure (C) (Table 3.2). This indicates the presence of electron transfer at the transition state rather than substrate bound intermediates for the oxidation of the substrates to the corresponding products.

Table 3.2. The percentage contribution of the molecular orbital fragments $\left(\mathrm{Mo}_{\mathrm{dxy}}\right)$ to HOMO and LUMO for substrate bound (B) and transition state structure (C) of formaldehyde and its chalcogen analog.

\begin{tabular}{lcccc}
\cline { 2 - 5 } Structure & \multicolumn{2}{c}{ B } & \multicolumn{2}{c}{ C } \\
\cline { 2 - 5 } & HOMO & LUMO & HOMO & LUMO \\
\hline ACT-FA & 3.39 & 55.07 & 20.57 & 37.86 \\
ACT-THIO FA & 3.28 & 47.48 & 21.72 & 31.51 \\
ACT-SELENO FA & 3.37 & 46.81 & 18.81 & 29.7 \\
\hline
\end{tabular}


The significant increase of $\% \mathrm{Mo}_{\mathrm{dxy}}$ to $\mathrm{HOMO}$ at the transition state structure (C) was due to the partial electron transfer from the substrates to the active site.

\subsubsection{Understanding the effect of chalcogen replacement on the interaction site using bond distance and bond order}

The bond distances of C-O, C-S, and C-Se bonds for free substrates and substratebound intermediates of formaldehyde and its chalcogen analogs were shown in (Figure $3.3)$.

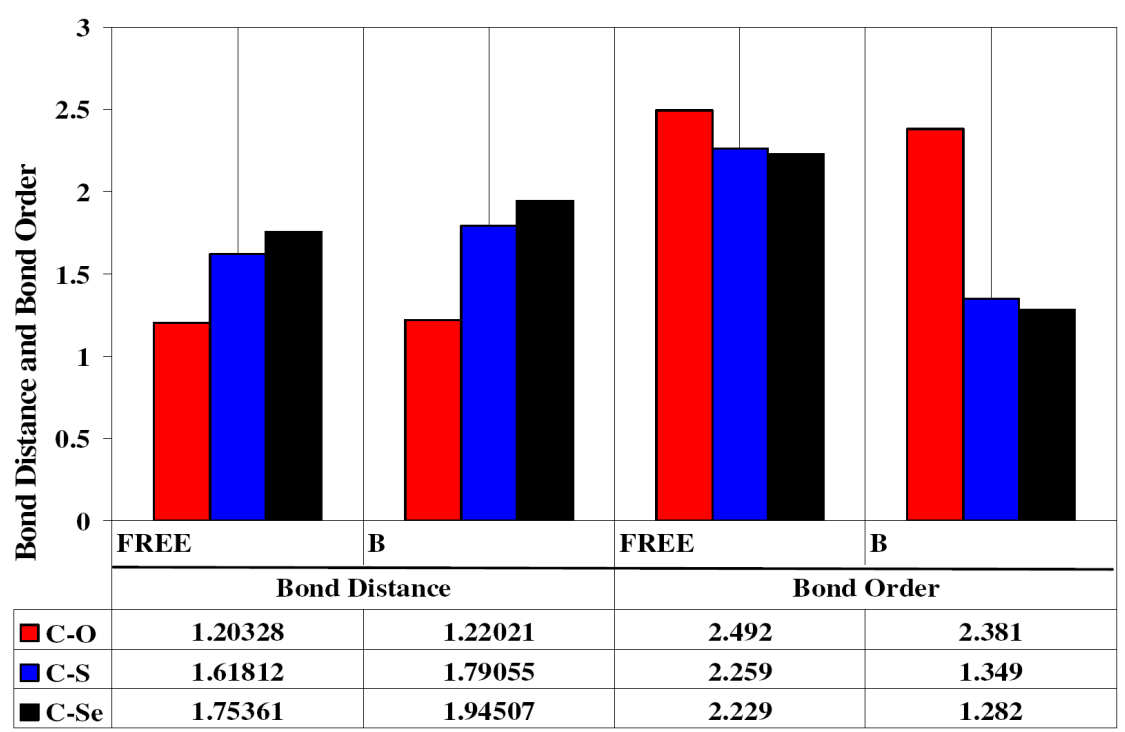

Figure 3.3. The bond distances and orders for $\mathrm{C}-\mathrm{O}, \mathrm{C}-\mathrm{S}$, and $\mathrm{C}-\mathrm{Se}$ bonds. The 1 st (red), 2nd (blue), and 3rd (black) bars (from left to right), respectively, indicated C-O, C-S, and C-Se bonds for substrate structures (FREE) and substrate-bound active site (ACT-FA, ACT-THIO FA, and ACT-SELENO FA) structure (B).

The C-S and C-Se bond distance of free substrates, respectively, were higher by $0.41484 \AA$ and $0.55033 \AA$ than the C-O bond. The C-S and C-Se bond distances for substrate bound intermediate, respectively, were higher by $0.57034 \AA$ and $0.72486 \AA$ than $\mathrm{C}-\mathrm{O}$ bond. This revealed a lengthening of C-O, C-S, and C-Se bonds for substrate bound intermediates relative to the free substrates due to the interaction of carbonyl carbon atom of the substrate to the catalytically liable equatorial oxygen of the active site. The bond order of $\mathrm{C}-\mathrm{O}, \mathrm{C}-\mathrm{S}$, and $\mathrm{C}-\mathrm{Se}$ bonds decreased in the reverse order of the 
bond distances. The decreasing of bond distances and an increase of bond order of chalcogen families (C-O, C-S, and C-Se) was due to the decreasing of electronegativity and increasing of polarizability down a group. As a result, the high electronegative oxygen atom was labile to interact with the electron deficient carbon atom than sulfur and selenium. Also, the higher polarization ability of selenium than oxygen and sulfur is due to its size and volume occupied by electrons. This occurs because of larger atoms have more loosely held electrons in contrast to smaller atoms with tightly bound electrons. Therefore, the variation in $\mathrm{C}-\mathrm{O}, \mathrm{C}-\mathrm{S}$, and $\mathrm{C}-\mathrm{Se}$ bonds is due to the electronegativity and polarization ability of chalcogen families.

\subsubsection{Understanding the effect of chalcogen replacement on the interaction site using frontier orbitals}

The maximum electron density is accommodated on the highest occupied molecular orbital (HOMO) than the lowest unoccupied molecular orbital (LUMO) of formaldehyde and its chalcogen analogs. As a result, the HOMO-LUMO interaction takes place between the HOMO of the substrate and LUMO of the active site for the formation of substrate-bound intermediates. In addition, the maximum electron density was accommodated along the dithiolene sulfur atom coordinated to the molybdenum metal for the substrate bound intermediate (B). This reveals the contribution of dithiolene sulfur atoms in electron transfer reactions. Moreover, the increasing of an electron density on the central metal (Mo) at the transition state structure (C) than substrate bound structure (B) indicates the partial electron transfer from the substrates to the active site as shown in Table 3.2 and Figure 3.4.

In summary, from the results obtained from electronic energy, Mulliken atomic charge, bond distance, bond order, and molecular orbital analysis, formaldehyde was more labile to interact to the reductive half-reaction active site of aldehyde oxidase as compared to thioformaldehyde and selenoformaldehyde. 


\begin{tabular}{|c|c|c|c|}
\hline Symbols & Structures & HOMO & LUMO \\
\hline \multirow{4}{*}{ FREE } & FA & & \\
\hline & THIO FA & & \\
\hline & SELENO FA & & \\
\hline & ACT-FA & & \\
\hline \multirow{2}{*}{ B } & ACT-THIO FA & & \\
\hline & ACT-SELENO FA & & \\
\hline \multirow{3}{*}{$\mathrm{C}$} & ACT-FA & & \\
\hline & ACT-THIO FA & & \\
\hline & ACT-SELENO FA & & \\
\hline
\end{tabular}

Figure 3.4. The frontier orbitals (HOMO and LUMO) for the free substrate, substratebound and transition state structures formaldehyde and its chalcogen analogs. 


\subsection{The effect of chalcogen replacement on the transition state structure: Prediction and characterization}

As described in the introduction section, the geometry of the transition state structure was central in understanding the course of the reaction mechanism, although the smallest fraction of the catalytic cycle was spent in the transition state. However, understanding how these events take place, in the presence of different substrates, was of considerable mechanistic interest. The geometries of formaldehyde and its chalcogen analog bound to the active site were optimized as described in the method section. The normalized energies were plotted as a function of reaction coordinates $(\mathrm{CH}, \mathrm{TS}$, and $\mathrm{SH})$ as shown in (Figure 3.5).

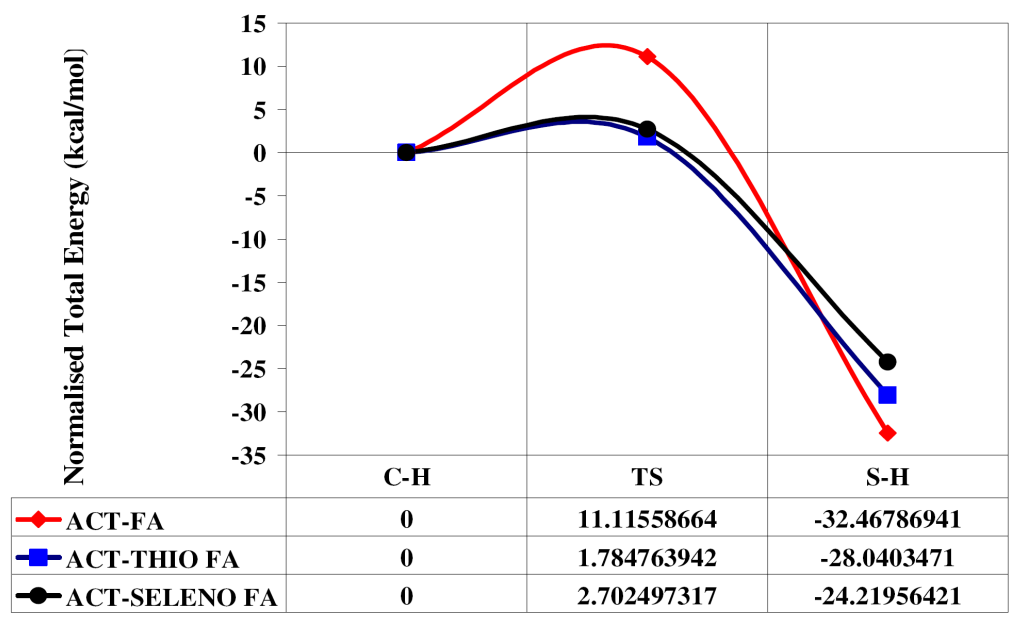

Figure 3.5. The normalized energies as a function of the reaction coordinates $(\mathrm{C}-\mathrm{H}, \mathrm{TS}$, and $\mathrm{S}-\mathrm{H})$. The symbols (C-H, TS, and S-H) represented, respectively, the pre-transition state close to the substrate bound $\left(\mathrm{Mo}^{\mathrm{VI}}-\mathrm{O}_{\mathrm{eq}} \mathrm{C}_{\mathrm{CRH}}\right)$, transition state $\left(\left[\mathrm{C}_{\mathrm{CRH}^{---}} \mathrm{H}_{\mathrm{RH}^{--}}\right.\right.$ $\mathrm{S}_{\mathrm{Mo}}{ }^{\ddagger}$ ), and post-transition state close to the product bound ( $\mathrm{Mo}^{\mathrm{VI}}-\mathrm{O}_{\mathrm{eq}}-\mathrm{C}_{\mathrm{CR}}(-\mathrm{SH})$.

The activation energy required to form the transition state structure for ACT-FA was higher by $9.33 \mathrm{kcal} / \mathrm{mol}$ and $8.41 \mathrm{kcal} / \mathrm{mol}$, respectively, to ACT-THIO FA and ACTSELENO FA. The lower activation energy in the oxidative hydroxylation of ACT-THIO FA reflects the kinetic feasibility of THIO FA than SELENO FA and FA. However, FA exhibits lower energy than THIO FA and SELENO FA during the conversion of reactants to the corresponding products (Figure 3.5). As a result, the oxidative hydroxylation of formaldehyde by aldehyde oxidase yielded a thermodynamically stable 
product. For a chemical reaction to occur, the reactants were expected to supply with enough activation energy to enable the reaction to proceed forward, in turning reactants into products. However, the activation energy for ACT-THIO FA was lower related to ACT-FA. On the contrary, FA was thermodynamically stable as compared to remaining chalcogen analogs. This reflected the performance of the reactions (spontaneous reaction) due to the change in negative free energy change and large equilibrium constant for the formation of thermodynamically stable products. Even though the oxidation of formaldehyde required higher energy barrier, it was the favored and preferred substrate than thioformaldehyde and selenoformaldehyde. This was due to the higher activation energy for formaldehyde bound intermediate $(\mathrm{CH})$ that entered to the transition state. This enabled the reaction to proceed in the forward direction turning reactants into thermodynamically stable products [6].

As described in the method section (Figure 2.2), the frequency calculations on the transition state structures of ACT-FA, ACT-THIO FA, and ACT-SELENO FA were carried out to characterize stationary points as minima or transition states. The calculations were carried out to monitor the movement of $\mathrm{H}_{\mathrm{RH}}$ from $\mathrm{C}_{\mathrm{RH}}$ to $\mathrm{S}_{\mathrm{Mo}}$. The electronic structure description for the transition state structure was characterized by one negative imaginary negative frequency that represents the saddle point that distinguishes the minimum characterized by all other positive frequencies. The frequency of ACT-FA TS was higher than ACT-THIO FA TS and ACT-SELENO FA TS (Figure 3.6).

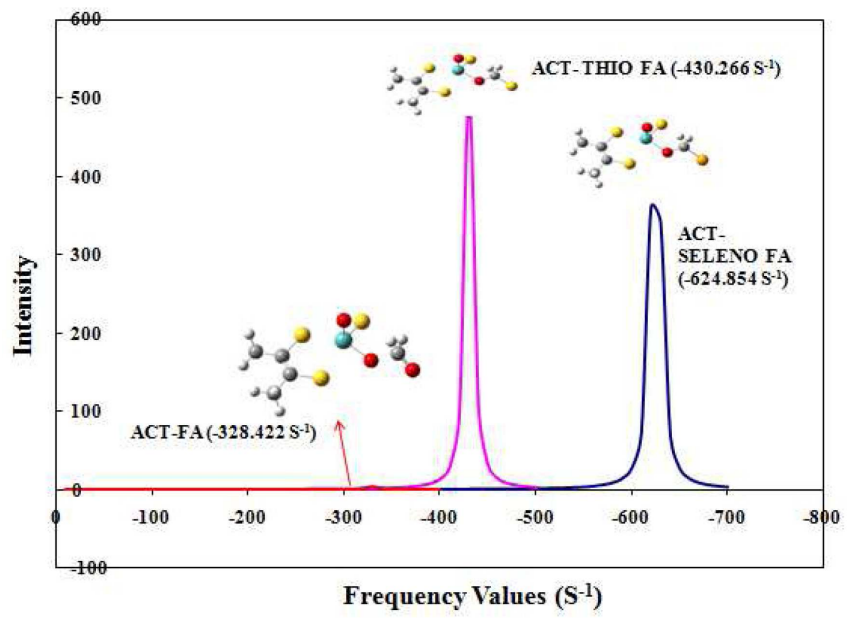

Figure 3.6. The plot of frequency values obtained from frequency calculations for the transition state (TS) structure. 
The highest the vibrational frequency of ACT-FA revealed that the time required to pass the barrier of the reaction was small as compared to ACT-THIO FA TS and ACTSELENO FA TS which enabled the faster reaction for the conversion of reactants to the corresponding products. However, the intensity (degree of vibrational frequency) of ACT-THIO FA TS was higher as compared to ACT-SELENO FA TS and ACT-FA TS (Figure 3.6). This phenomenon was correlated with the normalized energy profile shown in Figure 3.5 and revealed the kinetic feasibility of THIO FA compared to FA and SELENO FA.

In addition, the wave function description shows as the accommodation of maximum electron density along the dithiolene sulfur atoms of ACT-FA CH compared to ACTTHIO FA CH and ACT-SELENO FA CH. This was due to the overlap of $\pi$-orbital between $\mathrm{d}_{\mathrm{xy}}$-orbital of molybdenum and $\mathrm{p}_{\mathrm{y}}$-orbital of sulfur atoms. In addition, the electron density was highly accommodated at the carbonyl group of the substrate, particularly in the selenium ( $\mathrm{Se}$ ) and sulfur (S) substituted carbon group. The highest electron density was due to the ability of $\mathrm{Se}$ and $\mathrm{S}$ to polarize the electrons accommodated on the carbonyl carbon atom towards them. At the catalysis stage, there was a significant increase of $\% \mathrm{Mo}\left(\mathrm{d}_{\mathrm{xy}}\right)$ orbital and electron density on the catalytically labile equatorial oxygen $\left(\mathrm{O}_{\mathrm{eq}}\right)$ of the active site and carbonyl $\mathrm{C}$-atom $\left(\mathrm{C}_{\mathrm{RH}}\right)$ of the substrate Table 3.3.

Table 3.3. The percentage contribution of molybdenum $\left(\% \mathrm{Mo}_{\mathrm{dxy}}\right)$ for ACT-FA $\mathrm{SH}$, ACT-THIO FA SH, and ACT-SELENO FA SH.

\begin{tabular}{lccc}
\hline \multicolumn{4}{c}{$\boldsymbol{\% M o}_{\mathbf{d x y}}$} \\
Structure & $\mathbf{C H}$ & \multicolumn{1}{c}{ TS } & SH \\
\hline ACT-FA & 3.39 & 20.57 & 81.82 \\
ACT-THIO FA & 6.11 & 21.72 & 79.13 \\
ACT-SELENO FA & 6.58 & 18.81 & 76.99 \\
\hline
\end{tabular}

This enables the partial electron transfer towards the central metal (Mo) across the equatorial oxygen. This phenomenon was mainly observed in the catalysis stage of ACTFA TS) as shown in Table 3.3. However, at the product bound structures, the electron densities were shifted to the active site. The $\%$ Mo $\left(\mathrm{d}_{\mathrm{xy}}\right)$ for ACT-FA SH, ACT-THIO FA SH, and ACT-SELENO FA SH (Table 3.3), respectively, were increased by $61.25 \%$, 
$57.41 \%$ and $58.18 \%$ related to the transition state structures. On the other hand, there was higher $\%$ contribution of molybdenum on the transition state structure of ACT-FA as compared to ACT-THIO FA and ACT-SELENO FA due to the affinity of formaldehyde to react and the active sites ability to turn over into its product. The shifting of electron densities to the active site and the significant increasing of $\%$ Mo $\left(\mathrm{d}_{\mathrm{xy}}\right)$ predicted the transfer of electrons to the molybdenum center and hence the reduction of Mo from $\mathrm{Mo}^{\mathrm{VI}}$ to $\mathrm{Mo}^{\mathrm{IV}}$ as hydrogen migrated from $\mathrm{C}_{\mathrm{RH}}$ to the $\mathrm{S}_{\mathrm{Mo}}$ of the active site.

\section{Conclusion}

The replacement of chalcogen analogs (sulfur and selenium) on the carbonyl group of formaldehyde had a significant effect on the interaction site and transition state. Amongst the chalcogen substituted analogues, formaldehyde experienced higher energy at the initial stage of catalysis which made it labile to interact with the active site. The liability of formaldehyde enabled for the formation of Michaelis-Menten type complex and conversion to the energetically stable product. In general, the results obtained from total energy, Mulliken atomic charge, bonding description, and molecular orbital analyses, formaldehyde was favored and preferred as a tractable substrate to interact to the reductive half-reaction active site of aldehyde oxidase.

\section{Acknowledgment}

Author expresses his sincere gratitude to the Ministry of Education for giving the opportunity to study and financial support throughout the study. Author would like to thank Arba Minch University, Department of Chemistry for providing a safe environment and fulfilling the necessary materials for the completion of this research work.

\section{References}

[1] D.C. Pryde, D. Dalvie, Q. Hu, P. Jones, R.S. Obach and T. Tran, Aldehyde oxidase: an enzyme of emerging importance in drug discovery, J. Med. Chem. 53 (2010), 8441-8460. https://doi.org/10.1021/jm100888d

[2] S. Dastmalchi and M. Hamzeh-Mivehrod, Molecular modeling of human aldehyde oxidase and identification of the key interactions in the enzyme-substrate complex, $J$. Pharma. Sci. 13 (2005), 1-13. 
[3] G.I. Panoutsopoulos and C. Beedham, Kinetics and specificity of guinea pig liver aldehyde oxidase and bovine milk xanthine oxidase towards substituted benzaldehydes, Acta Biochimica. Polonica. 51(3) (2004), 649-663.

[4] J.M. Rebelo, J.M. Dias, R. Huber, J.J.G. Moura and M.J. Romao, Structure refinement of the aldehyde oxidoreductase from Desulfovibrio gigas (MOP) at $1.28 \AA$, J. Biol. Inorg. Chem. 6 (2001), 791-800. https://doi.org/10.1007/s007750100255

[5] Y. Yamaguchi, T. Matsumura, K. Ichida, K. Okamoto and T. Nishino, Human xanthine oxidase changes its substrate specificity to aldehyde oxidase type upon mutation of amino acid residues in the active site: Roles of active site residues in binding and activation of purine substrate, J. Biochem. 141 (2007), 513-524. https://doi.org/10.1093/jb/mvm053

[6] X.-H. Zhang and Y.-D. Wu, A theoretical study on the mechanism of the reductive halfreaction of xanthine oxidase, Inorg. Chem. 44 (2005), 1466-1471. https://doi.org/10.1021/ic0487301

[7] J.A. People, Gaussian, Inc., Pittsburgh, PA., 2003. Gaussian 03W Help Table of contents. (File:///D//worksoft/gaussian03/G03help/G03help/g03help.htm2003-12-3).

[8] S. Bell, T.J. Dines, B.Z. Chowdhry and R. Withnall, Computational chemistry using modern electronic structure methods, J. Chem. Edu. 84 (8) (2007), 1364-1370. https://doi.org/10.1021/ed084p1364

[9] C. Jacob, G.I. Giles, N.M. Giles and H. Sies, Sulfur and selenium: the role of oxidation state in protein structure and function, Angew. Chem. Int. Ed. 42 (2003), 4742-4758. https://doi.org/10.1002/anie.200300573

[10] P. Basu, J.F. Stolz and M.T. Smith, A coordination chemist's view of the active sites of mononuclear molybdenum enzymes, Rev. Art. 84(11) (2003), 1412-1419.

[11] P. Udhayakala and T.V. Rajendiran, Computational investigations on the corrosion inhibition efficiency of some pyridine based alkaloids, J. Chem. Bio. Phy. Sci. 2 (2011), 172-183. 